\title{
Un procedimiento alternativo al tradicional para la medición de alturas con clinómetro
}

\author{
Miguel Ángel López López ${ }^{1}$
}

\begin{abstract}
RESUMEN
Se presenta un procedimiento de medición indirecta de altura de árboles con clinómetro. El procedimiento permite evitar la medición de la pendiente y distancia horizontal entre el ojo del observador y el árbol objetivo. Se diseñó un experimento para comparar el método propuesto con el método tradicional en lo relativo a tiempos de medición y precisión de las mediciones. Los resultados del experimento indican que las alturas de árboles medidas mediante el método propuesto se correlacionan mejor que las alturas medidas por el método tradicional, con las alturas reales de los árboles. El tiempo requerido para la medición de un sitio es consistentemente, pero no significativamente menor con el método propuesto que con el método tradicional. Se concluye que el procedimiento propuesto implica ganancias desde los puntos de vista ergonómico, financiero y de precisión, durante el desarrollo de proyectos de inventario forestal.
\end{abstract}

PALABRAS CLAVE:

Altura de árboles, clinómetro, inventario forestal, medición forestal.

\begin{abstract}
A procedure for indirect measurement of tree height using clinometer is presented in this paper. The procedure allows avoiding the measurement of slope and horizontal distance between the observer and the objective tree. An experiment was designed to compare the proposed procedure with the traditional method in terms of time used for measurement and precision. The results from the experiment indicate that tree heights measured with the proposed method are better correlated with real tree heights than those obtained with the traditional method. The required time for measurement of a site using the proposed method is consistently, but not significantly lower than the time needed while using the traditional method. It is concluded that the new procedure implies gains from the ergonomic, financial, and precision viewpoints during the development of forest inventory projects.
\end{abstract}

KEY WORDS:

Tree height, clinometer, forest inventory, forest mensuration. 


\section{INTRODUCCIÓN}

No obstante que, de acuerdo con los expertos en inventarios forestales, una parte importante de los tiempos y costos del desarrollo de la parte operativa de un inventario forestal se debe a la localización de los sitios de muestreo en campo, el ahorro de tiempo durante las mediciones en un sitio puede disminuir de manera importante los costos totales del proyecto.

Aparentemente la medición de la altura de los árboles es una actividad sencilla que no presenta problemas significativos en campo. Sin embargo, existen especialistas en medición forestal que consideran que esta variable se puede convertir en un cuello de botella importante durante el levantamiento de un sitio forestal. De hecho, Chapman (1921) destaca que la medición de la altura de un árbol consume el mismo tiempo que la medición del diámetro a la altura del pecho de 10 árboles mediante una forcípula.

La medición de la altura de los árboles por medio del clinómetro, instrumento más comúnmente utilizado para este fin, mediante el método tradicional consume una parte muy significativa del tiempo empleado en la evaluación general de un sitio de muestreo durante las actividades de inventario forestal. En efecto, el procedimiento de medición de la altura implica al menos tres etapas, cada una de las cuales consume un tiempo considerable: 1) medición de la pendiente entre el árbol y el punto desde el cual se medirá la altura del árbol, 2) medición de la distancia horizontal entre el árbol y el punto desde el cual se medirá la altura del árbol y 3) toma de lecturas con el clinómetro.

El uso eficiente del equipo de medición puede contribuir de manera significativa al ahorro de tiempo y recursos económicos durante la etapa de mediciones de campo y proyectos de inventario forestal. En el presente documento se pone a consideración del lector un nuevo procedimiento para simplificar las mediciones de altura por medio del clinómetro o la pistola haga. Mediante este procedimiento es posible evitar las dos primeras etapas asociadas al procedimiento tradicional, por lo que se presume que se puede lograr un ahorro de tiempo y recursos financieros, así como un incremento de la precisión.

\section{PROCEDIMIENTO PROPUESTO}

El proceso que se plantea está basado principalmente en procedimientos algebraicos y de geometría analítica. Considérese el plano cartesiano de la Figura 1, En este plano, el eje de las abscisas (X) representa la distancia horizontal entre el árbol y el ojo del observador, mientras que el eje de las ordenadas $(Y)$ representa alturas tanto del árbol como de la regleta que se encuentra en la base del árbol, misma que tiene una longitud conocida. En este eje, lógicamente se ubica también la magnitud de la ordenada al origen.

Del ojo del observador a cualquier punto del árbol sólo existen líneas rectas, las cuales, si se ubican dentro de un plano cartesiano, pueden ser representadas cada una por una ecuación de la forma:

$Y=a X+b$

Donde:

$Y=$ Altura desde la base del árbol hasta cualquier punto en el fuste $(\mathrm{m})$. $a=$ Pendiente de la recta $(\%)$ 
$X=$ Distancia horizontal entre el árbol $y$ cualquier punto en la recta $(\mathrm{m})$

$b=$ Distancia vertical $(\mathrm{m})$ entre el origen del plano cartesiano (base del árbol) y el punto donde la recta interseca al eje de las ordenadas (ordenada al origen).

Dado que se conoce las dimensiones (altura) de la regleta (2 unidades en este caso) y que mediante el clinómetro es posible obtener la pendiente (\%) de la recta \#1, que en este caso es $-2 / 12=-0,167$ (dos unidades de desnivel en 12 unidades horizontales), entonces para esta recta se sabe que:

$Y_{1}=a_{1} X+b$, o sea, $Y_{1}=-0,167 X+2$

En esta ecuación, el valor de $a_{1}(-0,167)$ es la lectura de pendiente (\%) derivada del clinómetro. El valor (2) de $b_{1}$, la ordenada al origen, corresponde a la longitud de la regleta.

Análogamente, para la recta dos se tiene:

$Y_{2}=a_{2} X+b_{2}$, o sea, $Y_{2}=-0,333 X+0$

Hasta este momento, con la ayuda de la regleta y del clinómetro se ha obtenido las ecuaciones de las rectas \#1 y \#2. De acuerdo con la geometría analítica, la intersección de dos rectas está dada por la solución de sus ecuaciones, y las rectas \#1 y \#2 se intersecan en el ojo del observador. Por lo tanto, es posible determinar las coordenadas cartesianas del ojo del observador en el plano en estudio. Tales coordenadas se obtienen entonces al solucionar el sistema formado por

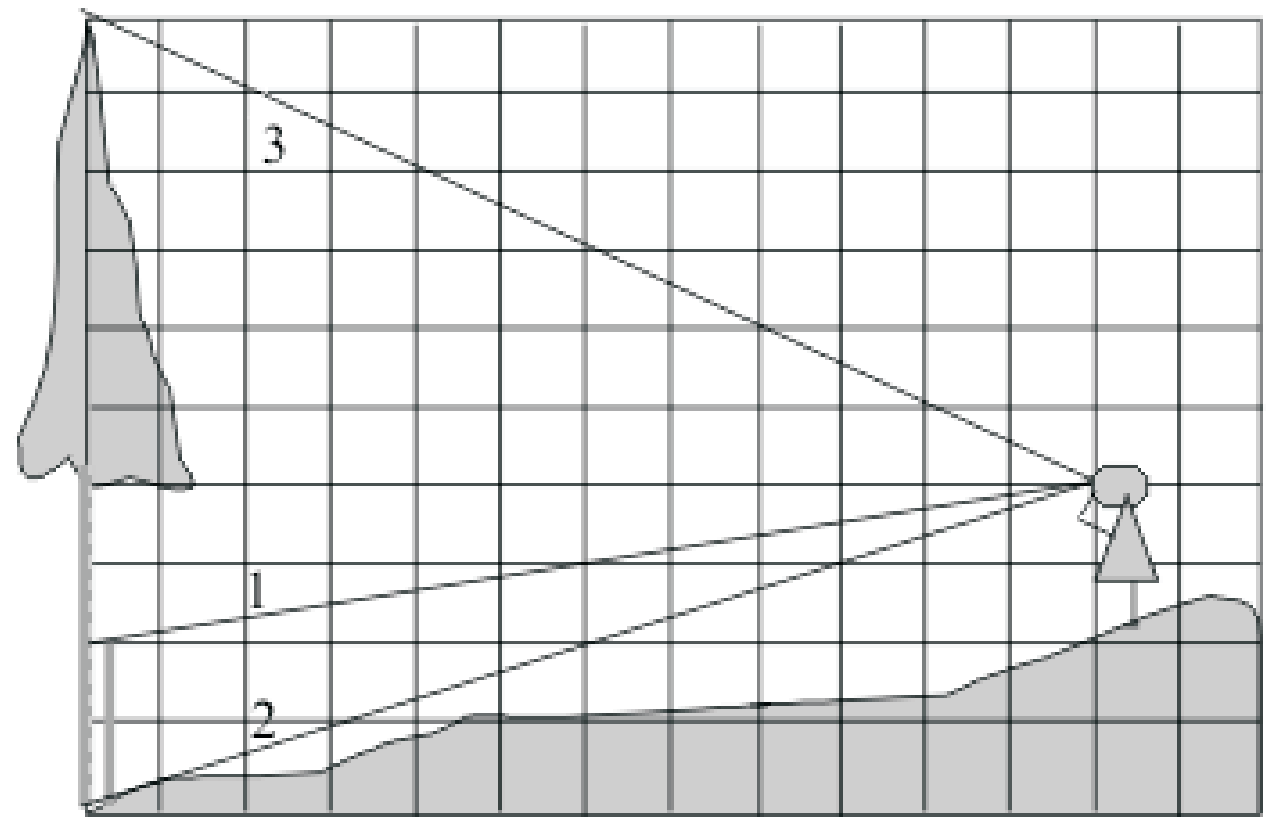

Fig. 1. Esquema general del procedimiento de medición de la altura de un árbol. 
las ecuaciones de las rectas \#1 y \#2, como se muestra a continuación:

$Y_{1}=-0,167 X+2$

$Y_{2}=-0,333 X+0$

$Y_{1}=Y_{2}$, puesto que ambas variables representan la altura a la que se encuentra el ojo del observador, dado que ahí coinciden ambas rectas.

\section{Sustituyendo $Y_{1}$ por $Y_{2}$ :}

$-0,333 X=-0,167 X+2$

Agregando 0,333X a ambos miembros de la ecuación:

$0=-0,167 x+0,333 x+2$

$0=0,166 X+2$

\section{Despejando $X$ :}

$X=-2 / 0,166=-12,0$

Sustituyendo el valor de $\mathrm{X}$ en la segunda ecuación:

$Y_{2}=-0,333^{*}-12=3,996 \sim 4$

La solución de este sistema de ecuaciones (el punto de intersección de estas dos rectas) produce un valor de $X=-12$ y $Y=$ 4. Es importante entender que estas son las coordenadas del ojo del observador. Sin embargo, más importante aún es comprender que ahora se conoce la distancia horizontal entre el observador y el árbol objetivo. Tal distancia es el valor del la coordenada $X$.

El cálculo del valor de $X$ ha sustituido a la medición de la pendiente en el método tradicional y a la medición de la distancia horizontal, la cual generalmente se realiza mediante el uso de una cuerda compensada por pendiente. Por otro lado, es fácil comprender que la medición en forma manual de la distancia horizontal (usando la cuerda compensada) conlleva una probabilidad de error mucho más alta que si se cuantifica mediante el uso de procedimientos geométrico-algebraicos. Por lo tanto, es muy probable que hasta este punto, por el nuevo procedimiento se tenga ya una ganancia en precisión.

Hasta este momento se ha solucionado una parte importante del problema de la determinación de la altura del árbol. Solamente resta conocer una parte de la ecuación de la recta \#3. Por analogía con las rectas \#1 y\#2, para la recta \#3 se tiene:

$Y_{3}=a_{3} X+b_{3}$

Puesto que ya se sabe que esta recta interseca a las otras dos en el ojo del

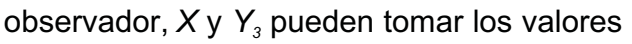
de sus coordenadas previamente determinados (-12 y 4). Por otro lado, $a_{3}$ (pendiente de la recta \#3 en \%) se obtiene dirigiendo el clinómetro hacia la punta del árbol. En la ecuación de la recta \#3 se tiene entonces sólo una incógnita, $b_{3}$, la cual corresponde precisamente a la altura del árbol. Sustituyendo los valores mencionados y despejando $b_{3}$ en la ecuación se obtiene $b_{3}=$ 10 unidades, como se muestra abajo:

$Y_{3}=a_{3} X+b_{3}$

Despejando $b_{3}$ :

$b_{3}=Y_{3} \quad a_{3} X$

Sustituyendo valores:

$b_{3}=4-(0,50 *-12)$

$b_{3}=10$ 


\section{PROGRAMAS DE CÓMPUTO}

\section{Para SAS (Statistical Analysis System)}

Si se prefiere, es posible tomar la información de campo en un formato que incluya las siguientes columnas:

El programa completo en SAS sería entonces:

Data a;

input an Isr lir Ipa;

longreg $=1,00 ; \quad$ altura $=($ longreg $/($ lir Isr) )*(lir+(Ipa*-1));

cards;

1201530

$220-2 \quad 25$

proc print; var an altura;

run;

Longreg significa longitud de la regleta (en metros). El valor $(1,00)$ de esta variable debe ser modificado cada vez que se use una regleta de diferente longitud.

Una vez que se corre este programa se tiene una salida en la ventana OUTPUT de $S A S$, misma que contiene dos columnas: el número de árbol y la altura del mismo.

\section{Para EXCEL}

Si se prefiere procesar los datos en EXCEL, debe registrarse las mismas variables que se levantan para el caso de $S A S$. Esta información debe entonces ser capturada en una hoja de EXCEL como se indica en la Figura 2.

El valor de la longitud de la regleta se asigna a

\begin{tabular}{cccc}
\hline $\begin{array}{c}\text { Identificación del } \\
\text { árbol }\end{array}$ & $\begin{array}{c}\text { Pendiente arriba de } \\
\text { la regleta }\end{array}$ & $\begin{array}{c}\text { Pendiente a la base } \\
\text { de la reg leta }\end{array}$ & $\begin{array}{c}\text { Pendiente a la } \\
\text { punta del árbol }\end{array}$ \\
\hline
\end{tabular}

En el programa SAS, estas variables pueden nombrarse como sigue:

\begin{tabular}{llll}
\hline$A N$ & $L S R(\%)$ & $L I R(\%)$ & $L P A(\%)$ \\
\hline
\end{tabular}

$A N=$ Arbol número, $L S R=$ Lectura a la parte superior de la regleta, $L I R=$ Lectura a la parte inferior de la regleta, y $L P A=$ Lectura a la punta del árbol. 
la celda B1, Una vez capturada toda la información como se muestra en la Figura 2, se hace click en la celda correspondiente a la altura del primer árbol (E3). Posteriormente se captura la siguiente secuencia:

$=(\$ B \$ 1 /(C 3-B 3)) *\left(C 3+\left(D 3^{*}-1\right)\right)$

Al presionar la tecla ENTER, aparece el valor correspondiente a la altura del primer árbol. Al copiar este valor en las celdas correspondientes a la altura de los demás árboles, automáticamente se calcula la altura de esos árboles de acuerdo con la información vertida en las columnas B, C y D.

\section{COMPARACIÓN DEL PROCEDIMIENTO PROPUESTO CON EL TRADICIONAL}

Con el objeto de contar con una primera prueba de las supuestas bondades del procedimiento de medición propuesto, se procedió a medir la altura de 25 árboles distribuidos en cinco sitios de cinco árboles cada uno. Las mediciones se hicieron usando el método tradicional, el procedimiento propuesto y por medio de una regla telescópica con capacidad para medir alturas hasta de $8,5 \mathrm{~m}$. Se registró además el tiempo que tomó la medición de las alturas por sitio con el método tradicional y con el nuevo procedimiento.

La Figura 3 muestra que las alturas de árboles medidas mediante el procedimiento propuesto se correlacionan mejor $(r=0,9170)$ que las realizadas mediante el método tradicional $(r=0,8950)$ con las alturas reales de los árboles. Por otro lado, una prueba de $t$ para comparar los dos métodos de medición de alturas en cuanto al tiempo necesario para evaluar los sitios indica que, aun cuando el método tradicional consumió invariablemente mayor tiempo (10,02 minutos/sitio) que el método propuesto (7,73 minutos/sitio), estadísticamente esta diferencia de tiempo no fue significativa $(p<0,07)$.

\section{DISCUSIÓN}

Durante el desarrollo de un programa de inventario forestal, el tiempo invertido en actividades de campo afecta

\begin{tabular}{c|cccccc}
\hline & A & B & C & D & E & F \\
\hline 1 & Longreg $=$ & 1,00 & & & & \\
\hline 2 & An & Lsr & Lir & Lpa & Altura & \\
\hline 3 & 1 & 20 & 15 & 30 & & \\
\hline 4 & 2 & 20 & -2 & 25 & \\
\hline 5 & & & & & \\
\hline
\end{tabular}

Fig. 2. Procedimiento de cálculo de la altura de árboles usando una hoja de Excel. 
significativamente los costos del proyecto. Si una cierta actividad puede realizarse en campo o bien en gabinete, su realización en gabinete por lo general resulta en un abatimiento de costos totales. Los altos costos del trabajo de campo derivan de las necesidades que por lo general tiene este tipo de actividades en cuanto al consumo de combustibles, pago de salarios a personal capacitado y pago de viáticos. Es por esta razón que cualquier esfuerzo que se haga por incrementar la eficiencia en esta clase de trabajos por lo general redunda en importantes ahorros económicos. En el caso del procedimiento presentado en este documento, la ganancia que se obtiene puede ser importante no solamente desde el punto de vista financiero, sino también de precisión en la información obtenida.

Desde el0 el procedimiento que ahora se propone, la medición de la altura de los árboles puede ser realizada por una sola persona, mientras que el método tradicional requiere de la participación de por lo menos dos personas para manejar la cuerda compensada. Esto significa que mientras una persona mide la altura de los árboles, la persona adicional que por el procedimiento tradicional estaría ocupada en la medición de la altura puede dedicarse a medir otras variables del sitio. Esto indudablemente redunda en un importante ahorro de tiempo y recursos económicos.

En el experimento que se estableció para comparar los métodos en relación al tiempo consumido para la medición de árboles se encontró que el ahorro de tiempo por el método propuesto no fue significativo, aunque sí fue consistente. El hecho de que en todos los sitios haya sido menor el tiempo consumido por el método propuesto que por el procedimiento tradicional indica que conforme incremente el número de árboles en el sitio, el ahorro de tiempo en la medición de cada árbol se acumulará, generando probablemente diferencias significativas entre los métodos. En este experimento se consideró solamente cinco árboles por sitio y se tuvo un ahorro promedio de 2,29 minutos mediante el procedimiento propuesto. Esto implica que en un sitio de 40 árboles, como los que comúnmente se encuentran en bosques de confieras, el ahorro pudiera ser del orden de 18,32 min. Un ahorro de esta magnitud en
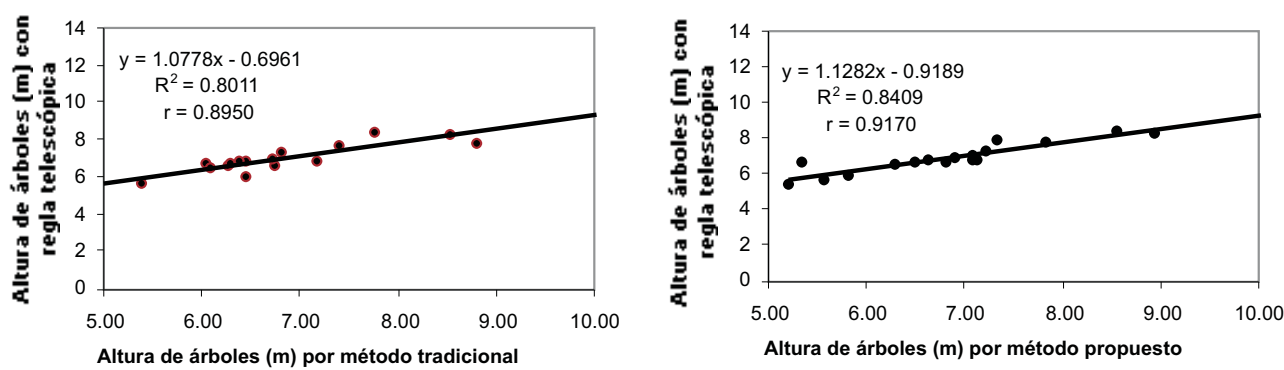

Fig. 3. Correlación de las alturas de árboles usando los métodos tradicional y propuesto, con las alturas medidas con regla telescópica 
cada sitio puede ser importante desde el punto de vista financiero, especialmente si se agrega a las otras ventajas potenciales del método planteado que se mencionaron arriba y que por restricciones financieras no fue posible incluir en el experimento, pudieran redundar en ahorros económicos considerables.

En términos de la precisión de la información recabada por ambos métodos, es probable que el procedimiento que aquí se ha desarrollado presente ventajas considerables sobre el procedimiento tradicional. Como ya se indicó anteriormente, el hecho de que en el sistema tradicional se tenga qué medir físicamente la distancia horizontal incrementa la probabilidad de que se cometan errores ya sea porque la pendiente del terreno no fue medida con toda precisión, porque la pendiente del terreno varía a lo largo de la distancia, porque se ha desviado la ruta debido a la presencia de un obstáculo insalvable (árbol, roca barranca, etc), o por cualquier otra razón. De hecho, Philip (1994) apunta que una de las mayores fuentes de error en la medición de alturas de árboles proviene de fallas en la medición de la distancia horizontal.

Debido a que el nuevo método evita el proceso completo de medición de la distancia horizontal (aunque ésta se estima en alguna parte del algoritmo de cálculos, valor de X), no existe la posibilidad de que se cometan esos errores. Sin embargo, para que pueda haber una ganancia real en la precisión de la estimación de la distancia horizontal y, consecuentemente en la estimación de la altura del árbol, se debe tener extremo cuidado en las mediciones de la pendiente hacia abajo y arriba de la regleta. El uso de un trípode es aconsejable para lograr una mayor precisión en las lecturas. Si no se tiene el cuidado suficiente en la toma de lecturas, se puede incurrir en estimaciones imprecisas de la distancia horizontal y por consiguiente, de la altura de los árboles.

Por otro lado, el método tradicional exige que el observador se ubique a una distancia determinada $(15,20,25 \mathrm{~m}$, etc., dependiendo del tipo de clinómetro que se use), la selección del punto de observación se vuelve entonces rigurosa (de acuerdo a la distancia horizontal predeterminada por el tipo de clinómetro usado) y puede resultar en una ubicación inferior a la óptima en términos de la visibilidad hacia la base del fuste o a la punta del árbol. El nuevo procedimiento, por el contrario, no presenta ninguna de estas limitaciones. La ubicación del punto de observación en este caso se realiza libremente, seleccionando el punto desde el cual se domine perfectamente la base del árbol, la parte superior de la regleta y la punta del árbol. La mejor visibilidad lograda puede indudablemente resultar en un incremento adicional de la precisión de la altura estimada. En resumen, se considera que debido a que la distancia horizontal se obtiene mediante cálculos y al hecho de que con el nuevo método se puede incrementar la visibilidad de los puntos a observar, la precisión de las mediciones puede ser incrementada sustancialmente. Es probable que la ventaja en precisión que se muestra en la Figura 3, sea una consecuencia de los puntos discutidos anteriormente.

\section{RECOMENDACIONES}

Se recomienda ampliamente ensayar el procedimiento descrito bajo condiciones reales durante el desarrollo de proyectos de inventario forestal y confrontarlo con el método tradicional con el objeto de verificar 
las supuestas bondades del sistema propuesto.

\section{REFERENCIAS}

Avery, T. E. 1967. Forest measurements. McGraw-Hill. Nueva York. 290 p.

Basurco, A. F. 1973. Apuntes de dasometría. Universidad Nacional-Inderena.
Escuela de Expertos Forestales. Medellín, Colombia. $121 \mathrm{p}$.

Chapman, H. H. 1921. Forest mensuration. 2da edición. J. Wiley and Sons. Nueva York. 557 p.

Philip, M. S. 1994. Measuring trees and forests. 2a. edición. CAB International. Reino Unido. 310 p. 